\title{
On the Cauchy-Schwarz inequality and its reverse in 2-*-semi inner product spaces
}

Received: 6 October 2017 / Accepted: 9 November 2018 / Published online: 24 November 2018

(C) The Author(s) 2018

\begin{abstract}
In this paper, we investigate the $\mathcal{A}$-valued Cauchy-Schwarz inequality and its reverse in a 2 -semi inner product $\mathcal{A}$-module over a locally $C^{*}$-algebra $\mathcal{A}$.
\end{abstract}

Mathematics Subject Classification $46 \mathrm{C} 50 \cdot 46 \mathrm{~L} 08$

\section{Introduction and preliminaries}

The concepts of 2-metric space and linear 2-normed space were first introduced by Gahler in 1963 and $1964[9,10]$. Since then, many authors, Freese et al. Gahler, Cho et al. Diminnie et al., and Gunawan et al. have developed extensively topological and geometric structures of 2-inner product spaces, 2-normed spaces, 2-metric spaces, semi-2-normed spaces, semi-2-metric spaces (see $[1,3-5,7-11,16]$ ). The term locally $C^{*}$ algebra was introduced by Inoue in 1971. Locally $C^{*}$-algebras are generalizations of $C^{*}$-algebras. Instead of being given by a single $C^{*}$-norm, the topology of a locally $C^{*}$-algebra is defined by a directed family of $C^{*}$-seminorms. The basic information about locally $C^{*}$-algebras can be found in $[6,12]$. The finitely generated modules equipped with inner products over some topological $*$-algebra and the standard Hilbert module $H_{A}$ over a locally $C^{*}$-algebra $\mathcal{A}$ were first considered by Mallios [14]. Most of the basic properties of Hilbert $C^{*}$-modules are still valid for Hilbert modules over locally $C^{*}$-algebras. Joita [13] explained the basic notions and theorems of the theory of Hilbert modules over a locally $C^{*}$-algebra. The Cauchy-Schwarz inequality plays an important role in the theory of inner product spaces (see, for instance, [20,21]), which is one of the classical inequalities. It is well known that in a 2 -semi inner product space $(X,\langle., . \mid\rangle$.$) , the Cauchy-Schwarz$ inequality will be formed as follows:

$$
|\langle x, y \mid z\rangle|^{2} \leq\langle x, x \mid z\rangle\langle y, y \mid z\rangle=\|x, z\|^{2}\|y, z\|^{2} \quad(x, y, z \in X)
$$

see [3]. Furthermore, the following additive reverse of the Cauchy-Schwarz inequality holds:

Theorem 1.1 [2, Theorem 1]. Let $(X,\langle., . \mid\rangle$.$) be a 2-inner product space on \mathbb{C}, x, y, z \in X$ and $a, b, \in \mathbb{C}$. If

$$
\operatorname{Re}\langle b y-x, x-a y \mid z\rangle \geq 0
$$

B. Mohebbi Najmabadi · T. L. Shateri ( $\varangle)$

Department of Mathematics and Computer Sciences, Hakim Sabzevari University, P.O. Box 397, Sabzevar, Iran

E-mail: t.shateri@hsu.ac.ir; t.shateri@gmail.com

B. Mohebbi Najmabadi

E-mail: behrozmohebbi1351@gmail.com; behruz_mohebbi@yahoo.com 
or equivalently

$$
\left\|x-\frac{a+b}{2} y\left|z\left\|\leq \frac{1}{2}|b-a|\right\| y, z \|\right.\right.
$$

holds, then we obtain

$$
\begin{aligned}
0 & \leq\|x, z\|^{2}\|y, z\|^{2}-|\langle x, y \mid z\rangle|^{2} \\
& \leq \frac{1}{4}|b-a|^{2}\|y, z\|^{4}-\left|\frac{a+b}{2}\|y, z\|^{2}-\langle x, y \mid z\rangle\right|^{2} \\
& \leq \frac{1}{4}|b-a|^{2}\|y, z\|^{4} .
\end{aligned}
$$

The constant $\frac{1}{4}$ is sharp in (1.3) in the sense that it cannot be replaced by a smaller constant.

The aim of the paper is to extend some inequalities from 2 -semi inner product spaces to 2-inner product $\mathcal{A}$ module spaces. In fact we generalize the above inequalities to a 2 -semi inner product $C^{*}$-module or a 2 -*-semi inner product such that the 2 -inner product map takes values in a locally $C^{*}$-algebra. First, we recall some definitions and basic properties of 2 -inner product spaces over locally $C^{*}$-algebras from $[15,17]$.

Definition 1.2 A $C^{*}$-seminorm on a topological $*$-algebra $\mathcal{A}$ is a seminorm $p$ such that $p(a b) \leq p(a) p(b)$ and $p\left(a a^{*}\right)=p(a)^{2}$ for all $a, b \in \mathcal{A}$. We denote by $S(\mathcal{A})$ the set of all continuous $C^{*}$-seminorms on $\mathcal{A}$.

Definition 1.3 A locally $C^{*}$-algebra is a Hausdorff complete complex topological $*$-algebra $\mathcal{A}$ whose topology is determined by all its continuous $C^{*}$-seminorms in the sense that a net $\left\{a_{i}\right\}_{i \in I}$ converges to 0 if and only if the net $\left\{p\left(a_{i}\right)\right\}_{i \in I}$ converges to 0 for all continuous $C^{*}$-seminorms $p$ on $\mathcal{A}$.

The following example is set by Philips [19]:

Example 1.4 [6] Consider the algebra $C[0,1]$ of all complex continuous functions on $[0,1]$. Let $\mathcal{S}$ be the family of all countable compact subsets of $[0,1]$. Endow $C[0,1]$ with the topology of uniform convergence on the elements of $\mathcal{S}$. In fact, the topology is defined by the family $\left\{p_{B}: B \in \mathcal{S}\right\}$ of the $C^{*}$-seminorms $p_{B}(f):=\left\|\left.f\right|_{B}\right\|_{\infty}$, for all $f \in C[0,1], B \in \mathcal{S}$. The algebra $C[0,1]$, equipped with this topology, is a locally $C^{*}$-algebra. Note that this topology is different from the supnorm topology of $C^{*}$-algebra $C[0,1]$. In fact, the topology cannot be normable.

Example 1.5 Every $C^{*}$-algebra is a locally $C^{*}$-algebra.

Definition 1.6 Let $\mathcal{A}$ be a locally $C^{*}$-algebra with unit. An element $a \in \mathcal{A}$ is positive and we write $a \geq 0$, if $a=a^{*}$ and $\operatorname{Sp}(a)=\{\lambda \mid a-\lambda 1$ is not invertible $\} \subseteq \mathbb{R}^{+}$. The set of all positive elements of $\mathcal{A}$ is denoted by $\mathcal{A}^{+}$. If $a, b \in \mathcal{A}$, then $a \leq b$ means that $b-a \in \mathcal{A}^{+}$.

Lemma 1.7 [13, Theorem 1.1.16]. Let $\mathcal{A}$ be a locally $C^{*}$-algebra. An element a in $\mathcal{A}$ is bounded if $\sup \{p(x) \mid p \in S(\mathcal{A})\}<\infty$. If the set of all bounded elements in $\mathcal{A}$ is denoted by $b(\mathcal{A})$, then the map $\|\cdot\|_{\infty}$ on $b(\mathcal{A})$ defined by $\|x\|_{\infty}=\sup \{p(x) \mid p \in S(\mathcal{A})\}$ is a $C^{*}$-norm on $\mathcal{A}$.

Remark 1.8 A $C^{*}$-algebra $\mathcal{A}$ can be embedded into a unital $C^{*}$-algebra $\tilde{\mathcal{A}}$ containing $\mathcal{A}$ as an ideal. The elements of $\tilde{\mathcal{A}}$ are pairs $(a, \lambda)$ with $a \in \mathcal{A}$ and $\lambda \in \mathbb{C}$; then we may assume that $\mathcal{A}$ is unital with identity 1. The center of $\mathcal{A}$ is denoted by $Z(\mathcal{A})$.

In the following, we recall the concept of a pre-Hilbert $\mathcal{A}$-module, where $\mathcal{A}$ is a locally $C^{*}$-algebra:

Definition 1.9 A pre-Hilbert $\mathcal{A}$-module is a complex vector space $E$ which is also a right $\mathcal{A}$-module, compatible with the complex algebra structure, equipped with an $\mathcal{A}$-valued inner product $\langle.,\rangle:. E \times E \rightarrow \mathcal{A}$ which is $\mathbb{C}$-linear and $\mathcal{A}$-linear in its second variable and satisfies the following relations:

$\left(I_{1}\right)\langle x, x\rangle \geq 0$ for every $x \in E$;

$\left(I_{2}\right)\langle x, y\rangle=\langle y, x\rangle^{*}$ for every $x, y \in E$;

$\left(I_{3}\right)\langle x, x\rangle=0$ if and only if $x=0$;

(I $\left.I_{4}\right)\langle x a, y b\rangle=a^{*}\langle x, y\rangle b$ for every $x, y \in E$ and $a, b \in \mathcal{A}$;

(I5) $\langle\alpha x+\beta y, z\rangle=\alpha\langle x, z\rangle+\beta\langle y, z\rangle$ for every $x, y, z \in E$ and $\alpha, \beta \in \mathbb{C}$. 
Example 1.10 Let $l^{2}(\mathcal{A})$ be the set of all sequences $\left\{a_{n}\right\}_{n \in \mathbb{N}}$ of elements of a locally $C^{*}$-algebra $\mathcal{A}$ such that the series $\sum_{n \in \mathbb{N}} a_{n} a_{n}^{*}$ is convergent in $\mathcal{A}$. Then $l^{2}(\mathcal{A})$ is a pre-Hilbert $\mathcal{A}$-module with respect to the pointwise operations and inner product is defined by

$$
\left\langle\left\{a_{n}\right\},\left\{b_{n}\right\}\right\rangle=\sum_{n \in \mathbb{N}} a_{n} b_{n}^{*} .
$$

Definition 1.11 Let $E$ be a right $\mathcal{A}$-module where $\mathcal{A}$ is a locally $\mathrm{C}^{*}$-algebra. An $\mathcal{A}$-combination of $x_{1}, x_{2}, \ldots, x_{n}$ in $E$ is written as follows:

$$
\sum_{i=1}^{n} x_{i} a_{i}=x_{1} a_{1}+x_{2} a_{2}+\cdots+x_{n} a_{n} \quad\left(a_{i} \in \mathcal{A}\right),
$$

and $x_{1}, x_{2}, \ldots, x_{n}$ are called $\mathcal{A}$-independent if the equation $x_{1} a_{1}+x_{2} a_{2}+\cdots+x_{n} a_{n}=0$ has exactly one solution, namely $a_{1}=a_{2}=\cdots=a_{n}=0$; otherwise, we say that $x_{1}, x_{2}, \ldots, x_{n}$ are $\mathcal{A}$-dependent.

The maximum number of elements in $E$, that are $\mathcal{A}$-independent, is called $\mathcal{A}$-rank of $E$.

Definition 1.12 Let $\mathcal{A}$ be a locally $C^{*}$-algebra and $E$ be a linear space by $\mathcal{A}$-rank greater than 1 , which is also a right $\mathcal{A}$-module. We define a function $\langle., . \mid\rangle:. E \times E \times E \rightarrow \mathcal{A}$ which satisfies the following properties:

$\left(T_{1}\right)\langle x, x \mid y\rangle=0$ if and only if $x=y a$ for $a \in \mathcal{A}$;

$\left(T_{2}\right)\langle x, x \mid y\rangle \geq 0$ for all $x, y \in E$;

$\left(T_{3}\right)\langle x, x \mid y\rangle=\langle y, y \mid x\rangle$ for all $x, y \in E$;

$\left(T_{4}\right)\langle x, y \mid z\rangle=\langle y, x \mid z\rangle^{*}$ for all $x, y, z \in E$;

$\left(T_{5}\right)\langle x a, y b \mid z\rangle=a^{*}\langle x, y \mid z\rangle b$ for all $x, y, z \in E$ and $a, b \in \mathcal{A}$;

(T6) $\langle\alpha x, y \mid z\rangle=\bar{\alpha}\langle x, y \mid z\rangle$ for all $x, y \in E$ and $\alpha \in \mathbb{C}$;

$\left(T_{7}\right)\langle x+y, z \mid w\rangle=\langle x, z \mid w\rangle+\langle y, z \mid w\rangle$ for all $x, y, z, w \in E$.

Then the function $\langle., . \mid$.$\rangle is called 2-*$ inner product and $(E,\langle., . \mid\rangle$.$) is called 2-*- inner product space. If E$ satisfies all conditions for a $2-*_{-}$-inner product except the second part of condition $\left(T_{1}\right)$, then we call $E$ is $2-*_{-}$ semi inner product space.

Example 1.13 Let $\mathcal{A}$ be an unital commutative locally $C^{*}$-algebra and $E$ be a pre-Hilbert $\mathcal{A}$-module with inner product $\langle\cdot, \cdot \cdot \cdot\rangle$. Define

$\langle\cdot, \cdot \mid \cdot\rangle: E \times E \times E \rightarrow \mathcal{A}$ by $(x, y, z) \longmapsto\langle x, y \mid z\rangle=\langle x, y\rangle\langle z, z\rangle-\langle x, z\rangle\langle z, y\rangle$.

Then $(E,\langle\cdot, \cdot \mid \cdot\rangle)$ is a $2-*$-semi inner product space.

Example 1.14 Let $\mathcal{A}$ be a commutative unital $C^{*}$-algebra. By the commutative Gelfand-Naimark theorem, we can identify $\mathcal{A}$ with $C(X)$, the algebra of continuous complex-valued functions on a compact Hausdorff space $X$. If $X$ was an Euclidean manifold, then one would analyse it by geometric techniques, among the most important of which is the study of vector bundles over $X$. A vector bundle $E$ can be described as follows: Take a fixed Euclidean space $H$ (note that, for example, if $(H,\langle\cdot, \cdot\rangle)$ is a Hilbert space a continuous 2-inner product which is defined on $H$ by $\left(\langle a, b \mid c\rangle=\langle a, b\rangle\|c\|^{2}-\langle a, c\rangle\langle b, c\rangle, a, b, c \in H\right)$ and for each $t$ in $X$ let $H_{t}$ be a subspace of $H$. Let $E$ be the space of all continuous functions $\xi$ from $x$ to $H$ such that, for all $t$ in $X, \xi(t) \in H_{t}$. Then $E$ is naturally endowed with a $C(X)$-valued 2-semi inner product. Namely, if $\xi, \eta, \gamma \in E$, then we define $\langle\xi, \eta \mid \gamma\rangle$ to be the function $t \longmapsto\langle\xi(t), \eta(t) \mid \gamma(t)\rangle_{H}$. Also, $E$ has the structure of a $C(X)$-module: given $\xi$ in $E$ and $f$ in $C(X)$, we define $\xi f$ to be the pointwise product $t \longmapsto \xi(t) f(t)$, which is an element of $E$.

The following theorems are proved in [17]:

Theorem 1.15 [17, Lemma 2.3, Theorem 2.4]. For every $x, y, z \in E$ and $\alpha \in \mathbb{C}$,

$$
\begin{aligned}
\text { (i) }\langle x, y \mid z\rangle= & \frac{1}{4}[\langle x+y, x+y \mid z\rangle-\langle x-y, x-y \mid z\rangle] \\
& +\frac{-i}{4}[\langle x+i y, x+i y \mid z\rangle-\langle x-i y, x-i y \mid z\rangle] . \\
\text { (ii) }\langle x, y \mid \alpha z\rangle= & |\alpha|^{2}\langle x, y \mid z\rangle . \\
\text { (iii) }\langle x, y \mid z a\rangle= & a^{*}\langle x, y \mid z\rangle a \quad(a \in \mathcal{A}) .
\end{aligned}
$$


Recall that

$$
\operatorname{Re}\langle x, y \mid z\rangle=\frac{1}{4}[\langle x+y, x+y \mid z\rangle-\langle x-y, x-y \mid z\rangle]
$$

and

$$
\operatorname{Im}\langle x, y \mid z\rangle=\frac{-1}{4}[\langle x+i y, x+i y \mid z\rangle-\langle x-i y, x-i y \mid z\rangle] .
$$

Theorem 1.16 [17, Theorem 2.6]. Let $(E,\langle\cdot, \cdot \mid \cdot\rangle)$ be a 2 -*-inner product space. For every $x, y, z \in E$ and $p \in S(\mathcal{A})$, the following inequality is satisfied:

$$
p(\langle x, y \mid z\rangle) \leq \sqrt{p(\langle x, x \mid z\rangle)} \sqrt{p(\langle y, y \mid z\rangle)}
$$

Proposition 1.17 [15, Proposition 1]. Let $(E,\langle., . \mid\rangle$.$) be a \mathcal{A}$-2-semi inner product space on a $C^{*}$-algebra $\mathcal{A}$. Then the following inequalities hold:

$$
\begin{aligned}
& (i)|\langle x, y \mid z\rangle|^{2}=\langle x, y \mid z\rangle^{*}\langle x, y \mid z\rangle \leq\|\langle x, x \mid z\rangle\|\langle y, y \mid z\rangle . \\
& (i i)\|\langle x, y \mid z\rangle\|^{2} \leq\|\langle x, x \mid z\rangle\|\|\langle y, y \mid z\rangle\|,
\end{aligned}
$$

for all $x, y, z \in E$.

Since $\langle x, x \mid z\rangle$ is a positive element in $\mathcal{A}$, there is a positive square root of $\langle x, x \mid z\rangle$ denoted by $|x, z|$ and for every $a \in \mathcal{A},|a|=\left(a^{*} a\right)^{1 / 2}$ and $\|x, z\|=\|\langle x, x \mid z\rangle\|^{1 / 2}$.

\section{The Cauchy-Schwarz inequality}

In this section we state and prove some results about the Cauchy-Schwarz inequality and its reverse in a 2-*semi inner product space.

Theorem 2.1 Let $\mathcal{A}$ be a locally $C^{*}$-algebra and $(X,\langle., . \mid\rangle$.$) be a 2$-*-semi inner product space. If $x, y, z \in X$ and $|x, z| \in Z(\mathcal{A})$, then

$$
|\langle x, y \mid z\rangle|^{2}=\langle x, y \mid z\rangle^{*}\langle x, y \mid z\rangle \leq|x, z|^{2}|y, z|^{2} .
$$

Proof For all $x, y, z \in X$ and $a \in \mathcal{A}$, we have

$$
0 \leq\langle x a-y, x a-y \mid z\rangle=a^{*}\langle x, x \mid z\rangle a-a^{*}\langle x, y \mid z\rangle-\langle y, x \mid z\rangle a+\langle y, y \mid z\rangle,
$$

which implies

$$
a^{*}\langle x, y \mid z\rangle+\langle y, x \mid z\rangle a \leq a^{*}\langle x, x \mid z\rangle a+\langle y, y \mid z\rangle .
$$

We know the positive number $\epsilon,|x, z|+\epsilon e$ is invertible [6]; take $a=\langle x, y \mid z\rangle(|x, z|+\epsilon e)^{-2}$, then we get

$$
2(|x, z|+\epsilon e)^{-2}|\langle x, y \mid z\rangle|^{2} \leq(|x, z|+\epsilon e)^{-4}|x, z|^{2}|\langle x, y \mid z\rangle|^{2}+|y, z|^{2} .
$$

Multiplying this by $(|x, z|+\epsilon e)^{2}$, using the fact that $|x, z|+\epsilon e \in Z(\mathcal{A})$ and applying [18, Theorem 2.2.5], we get

$$
2|\langle x, y \mid z\rangle|^{2} \leq(|x, z|+\epsilon e)^{-2}|x, z|^{2}|\langle x, y \mid z\rangle|^{2}+(|x, z|+\epsilon e)^{2}|y, z|^{2},
$$

that is

$$
|\langle x, y \mid z\rangle|^{2}-(|x, z|+\epsilon e)^{2}|y, z|^{2} \leq(|x, z|+\epsilon e)^{-2}|x, z|^{2}|\langle x, y \mid z\rangle|^{2}-|\langle x, y \mid z\rangle|^{2} .
$$

Multiplying $|x, z|^{2} \leq(|x, z|+\epsilon e)^{2}$ by $(|x, z|+\epsilon e)^{-1}\langle y, x \mid z\rangle$ from the left and by $(|x, z|+\epsilon e)^{-1}\langle x, y \mid z\rangle$ from the right, we get

$$
(|x, z|+\epsilon e)^{-2}|\langle x, y \mid z\rangle|^{2}|y, z|^{2} \leq|\langle x, y \mid z\rangle|^{2} .
$$


Taking this into account in (2.1), we have

$$
|\langle x, y \mid z\rangle|^{2}-(|x, z|+\epsilon e)^{2}|| y,\left.z\right|^{2} \leq 0,
$$

or

$$
|\langle x, y \mid z\rangle|^{2}-\left(|x, z|^{2}+2 \epsilon e|x, z|+\epsilon^{2} e\right)|| y,\left.z\right|^{2} \leq 0 .
$$

Thus, we conclude

$$
|\langle x, y \mid z\rangle|^{2}-|x, z|^{2}|| y,\left.z\right|^{2} \leq \epsilon(2|x, z|+\epsilon e)|y, z|^{2} .
$$

Letting $\epsilon \rightarrow 0$, we finally obtain $|\langle x, y \mid z\rangle|^{2} \leq|x, z|^{2}|y, z|^{2}$.

We get the following corollaries from Theorem 2.1.

Corollary 2.2 Let $\mathcal{A}$ be a commutative locally $C^{*}$-algebra, $(X,\langle., . \mid\rangle$.$) be a 2-*_{-}$semi inner product space, and $|x, z| \in b(\mathcal{A})$. Then we have

$$
p^{2}(\langle x, y \mid z\rangle) \leq p(\langle x, x \mid z\rangle) p(\langle y, y \mid z\rangle) \leq\|\langle x, x \mid z\rangle\|_{\infty} p(\langle y, y \mid z\rangle) .
$$

Corollary 2.3 Let $\mathcal{A}$ be a $C^{*}$-algebra, $(X,\langle., . \mid\rangle$.$) be a 2$-*-semi inner product space, and $|x, z| \in Z(\mathcal{A})$. Then we have

$$
|\langle x, y \mid z\rangle|^{2} \leq|x, z|^{2}|| y,\left.z\right|^{2} \leq\||x, z|\|^{2}|y, z|^{2} .
$$

Proof It is well known that $a^{*} b^{*} b a \leq\|b\|^{2} a^{*} a$ for all $a, b \in \mathcal{A}$ [18, Theorem 2.2.5]. In particular, if $|x, z| \in Z(\mathcal{A})$, then $|x, z|^{2}|y, z|^{2}=|y, z||x, z|^{2}|y, z| \leq\||x, z|\|^{2}|y, z|^{2}$.

Corollary 2.4 Let $\mathcal{A}$ be a $C^{*}$-algebra and $(X,\langle., . \mid\rangle$.$) be a 2$-*-inner product space. Then for $x, y, z \in X$ and $a \in \mathcal{A}$ we have

$$
|\langle x, y \mid z\rangle|^{2}=\||x, z|\|^{2}|y, z|^{2} \text { if and only if } y=\frac{1}{\|x, z\|^{2}} x\langle x, y \mid z\rangle+z a .
$$

Proof We may assume that $\|x, z\|=1$. First let us $y=x\langle x, y \mid z\rangle+z a$. Then suppose that $\langle y, x \mid z\rangle\langle x, y|$ $z\rangle=\langle y, x \mid z\rangle\langle x, x\langle x, y \mid z\rangle+z a \mid z\rangle=\langle y, x \mid z\rangle\langle x, x \mid z\rangle\langle x, y \mid z\rangle$, which implies that

$$
\begin{aligned}
0 & =\langle x\langle x, y \mid z\rangle+z a-y, x\langle x, y \mid z\rangle+z a-y \mid z\rangle \\
& =\langle y, x \mid z\rangle\langle x, x \mid z\rangle\langle x, y \mid z\rangle-\langle y, x \mid z\rangle\langle x, y \mid z\rangle+\langle y, y \mid z\rangle .
\end{aligned}
$$

Hence,

$$
\langle y, x \mid z\rangle\langle x, y \mid z\rangle=\langle y, y \mid z\rangle .
$$

Conversely, suppose that $\langle y, x \mid z\rangle\langle x, y \mid z\rangle=\langle y, y \mid z\rangle$. Then, we prove there exists $a \in \mathcal{A}$ such that $y=x\langle x, y \mid z\rangle+z a$. Since $\langle y, x \mid z\rangle\langle x, x \mid z\rangle\langle x, y \mid z\rangle \leq\|\langle x, x \mid z\rangle\|\langle y, x \mid z\rangle\langle x, y \mid z\rangle$, we have

$$
\begin{aligned}
0 & \leq\langle x\langle x, y \mid z\rangle-y, x\langle x, y \mid z\rangle-y \mid z\rangle \\
& =\langle y, x \mid z\rangle\langle x, x \mid z\rangle\langle x, y \mid z\rangle-\langle y, x \mid z\rangle\langle x, y \mid z\rangle-\langle y, x \mid z\rangle\langle x, y \mid z\rangle+\langle y, y \mid z\rangle \\
& \leq\langle y, x \mid z\rangle\langle x, y \mid z\rangle-\langle y, x \mid z\rangle\langle x, y \mid z\rangle=0 .
\end{aligned}
$$

Thus, there exists $a \in \mathcal{A}$ such that $y=x\langle x, y \mid z\rangle+z a$.

Now, we give the reverse of the Cauchy-Schwarz inequality. 
Theorem 2.5 Let $\mathcal{A}$ be a locally $C^{*}$-algebra and $(X,\langle., . \mid\rangle$.$) be a 2$-*- semi inner product space. If $a \in \mathcal{A}$ and $x, y, z \in X$ are such that $|x, z| \in Z(\mathcal{A})$ and

$$
\operatorname{Re}\langle y-x a, x b-y \mid z\rangle \geq 0
$$

holds, then we have the inequality

$$
\begin{aligned}
0 \leq|x, z|^{2}|y, z|^{2}-|\langle x, y \mid z\rangle|^{2} & \leq \frac{1}{4}|x, z|^{4}|b-a|^{2}-|| x,\left.z\right|^{2}(a+b) / 2-\left.\langle x, y \mid z\rangle\right|^{2} \\
& \leq \frac{1}{4}|x, z|^{4}|b-a|^{2} .
\end{aligned}
$$

Proof From Theorem 1.15 we have

$$
\Re\langle y-x a, x b-y \mid z\rangle=\frac{1}{4}(\langle z, z \mid x(b-a)\rangle-\langle z, z \mid 2 y-x(a+b)\rangle) \geq 0 .
$$

Then

$$
\begin{aligned}
\langle y-x(a+b) / 2, y-x(a+b) / 2 \mid z\rangle & \leq \frac{1}{4}(b-a)^{*}\langle z, z \mid x\rangle(b-a) \\
& =\frac{1}{4}|b-a|^{2}|x, z|^{2} .
\end{aligned}
$$

This is equivalent to

$$
|y, z|^{2} \leq \frac{1}{4}|b-a|^{2}|x, z|^{2}-\langle y, x \mid z\rangle \frac{a+b}{2}-\frac{a^{*}+b^{*}}{2}\langle x, y \mid z\rangle-\frac{|a+b|^{2}}{4}|x, z|^{2} .
$$

Using $|x, z| \in Z(\mathcal{A})$ and [18, Theorem 2.2.5], we get

$$
\begin{aligned}
|x, z|^{2}|y, z|^{2}-|\langle x, y \mid z\rangle|^{2} \leq & \frac{1}{4}|b-a|^{2}|x, z|^{4}+|x, z|^{2}\langle y, x \mid z\rangle \frac{a+b}{2}+\frac{a^{*}+b^{*}}{2}|x, z|^{2}\langle x, y \mid z\rangle \\
& -\frac{|a+b|^{2}}{4}|x, z|^{4}-|\langle x, y \mid z\rangle|^{2} \\
= & \frac{1}{4}|b-a|^{2}|x, z|^{4}-\left(|x, z|^{4} \frac{|a+b|^{2}}{4}-\frac{a^{*}+b^{*}}{2}|x, z|^{2}\langle x, y \mid z\rangle\right. \\
& \left.-\frac{a+b}{2}|x, z|^{2}\langle y, x \mid z\rangle+|\langle x, y \mid z\rangle|^{2}\right) \\
= & \frac{1}{4}|b-a|^{2}|x, z|^{4}-\left(\frac{1}{2}|x, z|^{2}(a+b)-\langle x, y \mid z\rangle\right)^{*}\left(\frac{1}{2}|x, z|^{2}(a+b)-\langle x, y \mid z\rangle\right) \\
= & \frac{1}{4}|b-a|^{2}|x, z|^{4}-\left|\frac{1}{2}\right| x,\left.z\right|^{2}(a+b)-\left.\langle x, y \mid z\rangle\right|^{2} \\
\leq & \frac{1}{4}|b-a|^{2}|x, z|^{4} .
\end{aligned}
$$

Remark 2.6 Let $x, z \in X$ such that $|x, z| \in Z(\mathcal{A})$ and let $a, b \in \mathcal{A}$ such that $a \neq b$. Assume that there exists $w \in X$ satisfying $|w, z|=|x, z|$ and $\langle x, w \mid z\rangle=0$. Let

$$
y=x\left(\frac{a+b}{2}\right)+w\left(\frac{b-a}{2}\right) .
$$

Then

$$
\begin{aligned}
|y, z|^{2} & =\left(\frac{a+b}{2}\right)^{*}|x, z|^{2}\left(\frac{a+b}{2}\right)+\left(\frac{b-a}{2}\right)^{*}|w, z|^{2}\left(\frac{b-a}{2}\right) \\
& =\frac{1}{4}|x, z|^{2}|a+b|^{2}+\frac{1}{4}|x, z|^{2}|b-a|^{2}
\end{aligned}
$$


and

$$
|\langle x, y \mid z\rangle|^{2}=\frac{1}{4}|x, z|^{4}|a+b|^{2}
$$

Thus

$$
|x, z|^{2}|y, z|^{2}-|\langle x, y \mid z\rangle|^{2}=\frac{1}{4}|x, z|^{4}|b-a|^{2}
$$

Hence, the constant $\frac{1}{4}$ in Theorem 2.11 is the best choice.

Remark 2.7 Let $(\mathcal{H},\langle., . \mid\rangle$.$) be a 2$ - semi inner product space on $\mathbb{C}$. Then, $|x, z|=\|x, z\|$ for all $x, z \in \mathcal{H}$ and if $\operatorname{dim} \mathcal{H}>2$, then Theorem 2.11 yields Theorem 2.11. The proof of Theorem 2.11 is simpler than the given proof of Theorem 2.10 in [2].

We come into the following conclusion that provides the property for the constant in the Cachy-Schwarz inequality:

Theorem 2.8 Let $\mathcal{A}$ be a locally $C^{*}$-algebra and $(X,\langle., . \mid\rangle$.$) be a 2-*_{-}$semi inner product space. If $\lambda \in \mathcal{A}$ and $x, y, z \in X$ are such that $\lambda,|x, z| \in Z(\mathcal{A})$, then

$$
|x, z|^{2}|y, z|^{2}-|\langle x, y \mid z\rangle|^{2}=|x-y \lambda, z|^{2}|y, z|^{2}-|\langle x-y \lambda, y \mid z\rangle|^{2} .
$$

Proof By properties of 2-*-inner product, it follows that, for any $\lambda,|x, z| \in Z(\mathcal{A})$,

$$
\begin{aligned}
|x-y \lambda, z|^{2}|y, z|^{2}-|\langle x-y \lambda, y \mid z\rangle|^{2}= & \langle x-y \lambda, x-y \lambda \mid z\rangle\langle y, y \mid z\rangle \\
& -(\langle y, x \mid z\rangle-\langle y, y \mid z\rangle \lambda)\left(\langle x, y \mid z\rangle-\lambda^{*}\langle y, y \mid z\rangle\right) \\
= & |x, z|^{2}|y, z|^{2}-2|y, z|^{2} \operatorname{Re}(\langle x, y \mid z\rangle \lambda)-|\lambda|^{2}|y, z|^{4} \\
& -|\langle x, y \mid z\rangle|^{2}+2|y, z|^{2} \operatorname{Re}(\langle x, y \mid z\rangle \lambda)+|\lambda|^{2}|y, z|^{4} \\
= & |x, z|^{2}|y, z|^{2}-|\langle x, y \mid z\rangle|^{2} .
\end{aligned}
$$

This completes the proof.

Corollary 2.9 Let $\mathcal{A}$ be a locally $C^{*}$-algebra and $(X,\langle., . \mid\rangle$.$) be a 2$-*-semi inner product space. If $\lambda \in \mathcal{A}$ and $x, y, z \in X$ are such that $\lambda,|x, z| \in Z(\mathcal{A})$, then

$$
|x, z|^{2}|y, z|^{2}-|\langle x, y \mid z\rangle|^{2} \leq|x-y \lambda, z|^{2}|y, z|^{2} .
$$

The equalities in 2.2 hold if and only if $\langle x, y \mid z\rangle=\lambda^{*}\langle y, y \mid z\rangle$.

For two parameters, we can have the following:

Theorem 2.10 Let $\mathcal{A}$ be a locally $C^{*}$-algebra and $(X,\langle., . \mid\rangle$.$) be a 2$-*- semi inner product space. If $\lambda, \mu \in \mathcal{A}$ and $x, y, z \in X$ are such that $\lambda, \mu,|x-y \lambda, z| \in Z(\mathcal{A})$, then

$$
\left(|x, z|^{2}|y, z|^{2}-|\langle x, y \mid z\rangle|^{2}\right)|\mu-\lambda|^{2}=|x-y \lambda, z|^{2}|x-y \mu, z|^{2}-|\langle x-y \lambda, x-y \mu \mid z\rangle|^{2} .
$$

Proof Take $w:=x-y \lambda$. Using some properties of a 2-*-inner product, we have

$$
\begin{aligned}
|\langle x-y \lambda, x-y \mu \mid z\rangle|^{2}= & \langle w+y(\lambda-\mu), w \mid z\rangle\langle w, w+y(\lambda-\mu) \mid z\rangle \\
= & \left(\langle w, w \mid z\rangle+(\lambda-\mu)^{*}\langle y, w \mid z\rangle\right)(\langle w, w \mid z\rangle+\langle y, w \mid z\rangle(\lambda-\mu)) \\
= & |w, z|^{4}+|w, z|^{2} \operatorname{Re}(\langle y, w \mid z\rangle(\lambda-\mu))+|\lambda-\mu|^{2}|w, z|^{2}|y, z|^{2} \\
& -|\lambda-\mu|^{2}\left(|w, z|^{2}|y, z|^{2}-|\langle w, y \mid z\rangle|^{2}\right) .
\end{aligned}
$$


Also, we notice that

$$
\begin{aligned}
\mid w & ,\left.z\right|^{4}+|w, z|^{2} \operatorname{Re}(\langle y, w \mid z\rangle(\lambda-\mu))+|\lambda-\mu|^{2}|w, z|^{2}|y, z|^{2} \\
& =|w, z|^{2}\left(|w, z|^{2}+2 \operatorname{Re}(\langle y, w \mid z\rangle(\lambda-\mu))+|\lambda-\mu|^{2}|y, z|^{2}\right) \\
& =|w, z|^{2}\left(|w+y(\lambda-\mu), z|^{2}\right) \\
& =|x-y \lambda, z|^{2}|x-\mu y, z|^{2} .
\end{aligned}
$$

Therefore, from (2.5) and (2.4), we obtain the desired result. This completes the proof.

Corollary 2.11 Let $\mathcal{A}$ be a locally $C^{*}$-algebra and $(X,\langle., . \mid\rangle$.$) be a 2$ - $^{*}$ semi inner product space. If $\lambda, \mu \in \mathbb{C} e$ and $x, y, z \in X$ are such that $|x-y \lambda, z| \in Z(\mathcal{A})$ and $\lambda \neq \mu$, then

$$
|x, z|^{2}|y, z|^{2}-|\langle x, y \mid z\rangle|^{2} \leq \frac{1}{|\mu-\lambda|^{2}}|x-y \lambda, z|^{2}|x-y \mu, z|^{2} .
$$

As an application of Theorem 2.10, we have the following:

Proposition 2.12 Let $\mathcal{A}$ be a commutative $C^{*}$-algebra and $(X,\langle., . \mid\rangle$.$) be a 2-*_{-}$semi inner product space. Then, for all $x, y, z, e \in X$ with $\langle e, e \mid z\rangle=1$ and $\lambda, \mu, \gamma, \eta \in \mathbb{C} e$ with $\lambda \neq \mu$ and $\gamma \neq \eta$, we have

$$
\|\langle x, y \mid z\rangle-\langle x, e \mid z\rangle\langle e, y \mid z\rangle\| \leq \frac{1}{|\lambda-\mu||\gamma-\eta|}(\|x-e \lambda, z\|\|x-e \mu, z\|\|x-e \gamma, z\|\|x-e \eta, z\|) .
$$

Proof We have $\langle x-\langle e, x \mid z\rangle e, y-\langle e, y \mid z\rangle e\rangle=\langle x, y \mid z\rangle-\langle x, e \mid z\rangle\langle e, y \mid z\rangle$,

$$
\mid\left\langle x-\langle e, x \mid z\rangle e,\left.z\right|^{2}=|x, z|^{2}-|\langle e, x \mid z\rangle|^{2}\right.
$$

and

$$
\mid\left\langle y-\langle e, y \mid z\rangle e,\left.z\right|^{2}=|y, z|^{2}-|\langle e, y \mid z\rangle|^{2} .\right.
$$

Applying the Cauchy-Schwarz inequality and Corollary 2.11, we have

$$
\begin{aligned}
\|\langle x, y \mid z\rangle-\langle x, e \mid z\rangle\langle e, y \mid z\rangle\| & \leq \|\langle x-\langle e, x \mid z\rangle e, z\|\mid\|\langle y-\langle e, y \mid z\rangle e, z \| \\
& =\left\||x, z|^{2}-|\langle e, x \mid z\rangle|^{2}\right\|^{1 / 2}\left\||y, z|^{2}-|\langle e, y \mid z\rangle|^{2}\right\|^{1 / 2} \\
& \leq \frac{1}{|\lambda-\mu||\gamma-\eta|}(\|x-e \lambda, z\|\|x-e \mu, z\|\|x-e \gamma, z\|\|x-e \eta, z\|) .
\end{aligned}
$$

Open Access This article is distributed under the terms of the Creative Commons Attribution 4.0 International License (http:// creativecommons.org/licenses/by/4.0/), which permits unrestricted use, distribution, and reproduction in any medium, provided you give appropriate credit to the original author(s) and the source, provide a link to the Creative Commons license, and indicate if changes were made.

\section{References}

1. Cho, Y.J.; Diminnie, C.R.; Freese, R.W.; Andalafte, E.Z.: Isosceles orthogonal triples in linear 2-normed spaces. Math. Nachr. 157, 225-234 (1992)

2. Cerone, P.; Cho, Y.J.; Dragomir, S.S.; Kim, S.S.: Refinements of some reverses of Schwarz inequality in 2-inner product spaces and applications for integrals. J. Indones. Math. Soc. 12(2), 185-199 (2006)

3. Cho, Y.J.; Lin, P.C.S.; Kim, S.S.; Misiak, A.: Theory of 2-Inner Product Spaces. Nova Science Publishes Inc, New York (2001)

4. Diminnie, C.; Ghler, S.; White, A.: 2-Inner product spaces II. Demonstr. Math. 1, 169-188 (1977)

5. Dragomir, S.S.; Cho, Y.J.; Kim, S.S.; Sofo, A.: Some Boas-Bellman type inequalities in 2-inner product spaces. J. Inequal. Pure Appl. Math. 6(2), 1-13 (2003)

6. Fragoulopoulou, M.: Topological Algebras with Involution. North Holand, Amsterdam (2005)

7. Freese, R.W.; Cho, Y.J.: Geometry of Linear 2- Normed Spaces. Nova Science Publishers Inc, New York (2001) 
8. Freese, R.W.; Gahler, S.: Remarks on semi-2-normed spaces. Math. Nachr. 105, 151-161 (1982)

9. Gahler, S.: 2-metriche raume und ihre topologische struktur. Math. Nachr. 26(1-4), 115-148 (1963)

10. Gahler, S.: Lineare 2-normierte raume. Math. Nachr. 28, 1-43 (1965)

11. Gozali, S.M.; Gunawan, H.: On b-orthogonality in 2-normed spaces. J. Indones. Math. Soc. 16, 127-132 (2009)

12. Inoue, A.: Locally $C^{*}$-algebras. Mem. Fac. Sci. Kyushu Univ. Ser. A 25, 197-235 (1971)

13. Joita, M.: Hilbert modules over locally $C^{*}$-algebras. Editura Universitatii din Bucuresti, Bucharest (2006)

14. Lance, C.: Hilbert $C^{*}$-Moudules, London Mathematical Society. Lecture Note Series 210. Cambridge University Press, Cambridge (1995)

15. Mehdiabad Mahchari, T.; Nazari, A.: 2-Hilbert $\mathrm{C}^{*}$-modules and some Gruss type inequalities in $\mathcal{A}$-2-inner product spaces. Math. Inequal. Appl. 18(2), 721-754 (2015)

16. Misiak, A.: n-Inner product spaces. Math. Nachr. 140(1), 299-319 (1989)

17. Mohebbi Najmabadi, B.; Shateri, T.L.: 2-Inner product which takes values on a locally $C^{*}$-algebra. Indian J. Math. Soc. $\mathbf{8 5}(1-2), 218-226(2018)$

18. Murphy, G.J.: $C^{*}$-Algebras and Operator Theory. Academic Press, Boston (1990)

19. Philips, N.C.: Inverse limits of $C^{*}$-algebras. J. Oper. Theory 19(1), 159-195 (1988)

20. Rassias, T.M.: Survey on Classical Inequalities. Kluwer Academic Publishers, Dordrecht (2000)

21. Rassias, T.M.: Functional Equations, Inequalities and Applications. Kluwer Academic Publishers, Dordrecht (2003)

Publisher's Note Springer Nature remains neutral with regard to jurisdictional claims in published maps and institutional affiliations. 